\title{
SPATIAL AND TEMPORAL COHERENCE IN OPTICAL PARAMETRIC DEVICES PUMPED WITH MULTIMODE BEAMS
}

\author{
V. Pašiškevičius ${ }^{a}$, V. Smilgevičius ${ }^{b}$, R. Butkus ${ }^{b}$, R. Coetzee ${ }^{a}$, and F. Laurell ${ }^{a}$ \\ ${ }^{a}$ Department of Applied Physics, Royal Institute of Technology, Roslagstullsbacken 21, 10691 Stockholm, Sweden \\ ${ }^{\mathrm{b}}$ Laser Research Center, Vilnius University, Sauletekio 10, 10223 Vilnius, Lithuania \\ Email:vp@kth.se
}

Received 16 January 2018; accepted 22 March 2018

\begin{abstract}
We investigate the emergence of spatial and temporal coherence for the fields in the noncritical nondegenerate parametric second-order down-conversion process pumped with low spatial and temporal coherence beams. It is shown that in this scenario, which is of considerable practical importance, the parametric gain in the near field breaks down into an ensemble of mutually incoherent beamlets containing parametric waves. The field generated in a single beamlet is fully spatially coherent. The size of such coherent parametric gain regions is governed by the near-field spatial coherence radius of the pump, which also acts as a parameter, restraining the linear diffraction of the parametric waves generated in the nonlinear interaction. Furthermore, we experimentally demonstrate how the spatial and temporal coherence can be substantially enhanced by manipulating the spatial field correlation of the multilongitudinal and multi-transversal mode pump.
\end{abstract}

Keywords: optical parametric generators and oscilliators, nonlinear optical ferroelectric structures, temporal and spatial photon correlations, spatial and temporal coherence

PACS: 42.65.Lm, 42.65.Yj, 42.70.Mp

\section{Introduction}

High-power and high-energy optical parametric devices such as optical parametric generators (OPGs), optical parametric oscillators (OPOs) and optical parametric amplifiers (OPAs) are, in many cases, pumped by highly multi-transversal mode Q-switched solid-state lasers. The most compelling reasons for using these pump lasers is their simplicity, efficiency, reliability and low cost. Although the output beams derived from such lasers are essentially non-diffraction-limited, showing large $\mathrm{M}^{2}$ values, the intensity distribution can approach a supergaussian or top-hat profile, which is thought to facilitate a more efficient pumping of parametric devices with high power conversion rates [1,2]. As high nonlinearity optical materials, such as periodically poled $\mathrm{LiNbO}_{3}$ (PPLN) and $\mathrm{KTiOPO}_{4}$ (PPKTP), are becoming available with large optical aperture sizes [3-6], these highpower and large- $\mathrm{M}^{2}$ beams can be employed for the output power scaling in nanosecond OPOs and OPAs.

It is well known that in nonlinear three-wave mixing (TWM) interactions the spatial and temporal degrees of freedom are closely coupled owing to the dispersive nature of the wavevectors of interacting fields $\mathbf{k}_{j}(\omega)$ and the essentially vectorial nature of the interaction. Here $j=\mathrm{p}, \mathrm{s}$, i denotes the pump, signal and idler waves, respectively. A typical ensemble-averaged observation in quantum-noise-seeded OPGs and OPOs is that the spatial mode divergence (proportional to the Fourier transform of the spatial first-order correlation) as well as the width of the intensity spectrum (proportional to the Fourier transform 
of the temporal first-order correlation) is monotonously increasing as the pump power is increased. It should be reminded that from the standpoint of classical theory the parametric gain is $\propto \operatorname{sinch}^{2}\left(\sqrt{\left(\Gamma^{2}-(|\Delta \mathbf{k}| / 2)^{2}\right) z}\right)$. As the nonlinear coupling $\Gamma$, proportional to the pump power, is increased, the gain will be exponentially expanding in spatial frequency as well as in temporal frequency $\omega$. Here, $\Delta \mathbf{k}=\mathbf{k}_{\mathrm{p}}(\omega)-\mathbf{k}_{\mathrm{s}}\left(\omega, \theta_{\mathrm{s}}\right)-\mathbf{k}_{\mathrm{i}}\left(\omega, \theta_{\mathrm{i}}\right)$, where $\theta_{\mathrm{s}}$ and $\theta_{\mathrm{i}}$ are the angles of the signal and the idler with respect to the pump, represents the vectorial phase mismatch which is responsible for the spatial-temporal coupling in TWM processes. The deterioration of the spatial and temporal coherence of the parametric waves is further exacerbated by pumping with partially coherent multi-longitudinal and multi-transversal mode beams [7], which have wavevectors $\mathbf{k}_{\mathrm{p}}(\delta \omega, \delta \theta)$ randomly distributed around the central frequency and the axial propagation direction. Such beams are typically produced by nanosecond high-energy Q-switched lasers.

The parametric gain is sensitive to the phases of interacting optical waves. The total phase of the interaction remains fixed, $\varphi_{\mathrm{p}}-\varphi_{\mathrm{s}}-\varphi_{\mathrm{i}}=-\pi / 2$, modulo $2 \pi$. However, the phase difference between the pump and the signal (or the idler) seeded by the quantum and thermal noise can drift without bounds [8]. Note that the phases of the signal and the idler under these restrictions can simultaneously change by $\pi$, which influences the state of the OPO self-locked at degeneracy [9]. Nonlinear, power-dependent phase jumps can also be produced by a cascaded TWM in parametric devices operating at high conversion efficiencies, which might result in further decrease in the temporal and spatial coherence of parametric beams [10]. In unseeded TWM the first parametric amplification step starts from the delta-correlated quantum noise, therefore some randomness is expected in the macroscopic parameters of the parametric device. Indeed, in the initial linearized quantum-optical theoretical treatments of OPA it was found that the output of the device can be broken down into an amplified coherent signal, zero-point fluctuations and amplified spontaneous and thermal emission, the latter mostly contributing to the bandwidth of the OPA, very similar to that in lasers [11, 12,. By developing a quantum-mechanical linearized OPA model, employing the Wigner quasi-probability distribution, Mollow and Glauber revealed increasingly strong signal-idler correlations during the ongoing process of parametric amplification [13]. Such correlations result in coherent state squeezing observed in TWM devices [14]. Augmenting the TWM quantum-mechanical model with the pump system revealed a crucial role played by the phase fluctuations of the pump [15] and triple correlations, i.e. correlations between the pump, signal and idler in the evolution of the quantummechanical Wigner function in OPAs and OPOs, even in nondegenerate TWM, i.e. when the signal and idler frequencies are different [16].

In some instances, however, the process of coherence loss can be mitigated, at least for one of the interacting waves. For instance, temporal coherence can be increased even for low-coherence pumping by convective separation of one of the waves in a strongly group-velocity-mismatched TWM [17]. The extreme convection is obtained in backward-wave parametric interactions where the backward-generated wave can acquire temporal coherence much higher than that of the pump [18-20]. Moreover, it was shown experimentally that the spatial coherence of the signal in a short-cavity (a large Fresnel number) singly-resonant OPO (SRO) can be drastically improved by employing pumping with Bessel-Gauss beams [21, 22]. The latter observation seems to indicate that the abovementioned spatial-temporal coupling inherent to the parametric process can be tailored.

Prompted by these observations, we experimentally investigated the emergence of the coherence properties in nondegenerate OPGs and OPOs pumped by multi-longitudinal and multitransversal mode beams. In particular, we show that in the near field the parametric beams consist of a seemingly stochastic distribution of perfectly spatially coherent beamlets propagating in slightly different directions and having different central frequencies. The spatial and spectral properties of these beamlets are primarily determined by the spatial and temporal correlations of the pump field, as well as the group-velocity mismatch between the interacting waves. The far field then consists of a largely incoherent summation of the radiation from these beamlets. Given this physical picture, it is clear that the usual 
classical nonlinear coupled wave equations for the field amplitudes cannot give a direct insight into the parametric generation process. As a simplest model which can give the necessary understanding, we introduce here nonlinear coupled equations for the first-order correlation functions, which by necessity contain triple correlations. Unfortunately, a quantum-mechanical model with sufficient complexity to reproduce the experimental observations does not exist today, to the best of our knowledge. It would be interesting to develop such theory, considering that the behaviour of triple correlations has been proposed as a Yes/No test of quantum mechanics with respect to stochastic electrodynamics, and other hidden variable theories [16]. Finally, based on the insights gleaned from the above experiments, we demonstrate that manipulation of the spatial correlation properties of the pump beam can be used for generating diffraction-limited parametric beams, even in a simple OPG, without any help by a resonant cavity for selecting the transversal modes.

This paper is organized as follows: we first present the experimental evidence of the gain channel breakdown into beamlets in the OPG pumped by a multimode beam and discuss the physical mechanism behind this phenomenon. Then we will elucidate our theory by experimental investigation of the coherence properties of the near and far fields generated in the multimode OPG and OPO. Finally, the experimental demonstration is given of how the spatial coherence properties of parametric beams can be controlled by manipulating the spatial correlations of the pump field, including pumping with Bessel-Gauss beams.

\section{Coherence properties of multimode-pumped OPG}

\subsection{Experiment}

Before proceeding with investigation of the farfield properties of the parametric beams generated with multi-transversal and multi-longitudinal mode pumping, we first investigated the nearfield properties in a traveling-wave nanosecond OPG. The near field of the OPG reflects the structure of the parametric gain channel, which eventually defines the far-field coherence properties and the brightness of the parametric beam. In an OPO, the parametric superfluorescence is recycled in the cavity and acts as a seed for subsequent roundtrips. So the statistical properties of the OPO-generated field will stem from those in OPG with an additional spatial and temporal integration within the cavity. In order to generate the substantial parametric superfluorescence using nanosecond pulses under relatively loose focusing conditions, we employed a $12 \mathrm{~mm}$-long PPKTP crystal. The PPKTP had a domain inversion period of $12.77 \mu \mathrm{m}$ and was designed for generation of a signal around $650 \mathrm{~nm}$ at room temperature, when pumped by a frequency doubled $\mathrm{Nd}$ :YAG laser at $532 \mathrm{~nm}$. The corresponding idler wavelengths were around $2.93 \mu \mathrm{m}$. The PPKTP crystal was fabricated from a flux-grown KTP wafer by using the standard electric-field poling technique [23]. The pump was an actively Q-switched, flashlamp pumped Nd:YAG laser (New Wave Research Minilase I) which, when frequency doubled, generated 5 ns pulses with energy up to $15 \mathrm{~mJ}$. The laser output beam was far from diffraction-limited with a measured $\mathrm{M}^{2}$ of about 7. The pump beam was loosely focused with an $f=500 \mathrm{~mm}$ lens to the FWHM radius $w=113 \mu \mathrm{m}$ in the PPKTP crystal, corresponding to a confocal length of about $22 \mathrm{~mm}$ in PPKTP.

The near field formed by the parametric signal and the pump were analysed for each shot by imaging the output plane of the PPKTP crystal onto the plane of a high-frame-rate CCD camera (DALSA-CA-D1). The imaging lens $(f=100 \mathrm{~mm})$ was placed $113 \mathrm{~mm}$ from the PPKTP exit surface, which resulted in a magnification of 7.45. The pump intensity distribution in the focus is shown in Fig. 1. The beam in the focus is clearly non-Gaussian and astigmatic, due to the properties of thermal lensing in the pump laser cavity. In the far field, on the other hand, the pump intensity distribution could be fit to the Gaussian with 95\% confidence. The intensity distribution in the near field contains characteristic non-uniformities, as could be seen in Fig. 1. The mean FWHM nonuniformity diameter of $40 \mu \mathrm{m}$ (standard deviation of $8 \mu \mathrm{m}$ ) has been obtained by processing 100 independent CCD frames corresponding to different pump pulses. The mean amplitude of the relative pump spatial intensity variation in the ripples was 0.18 (standard deviation 0.08). Although the mode structure of the near-field pump is not well 

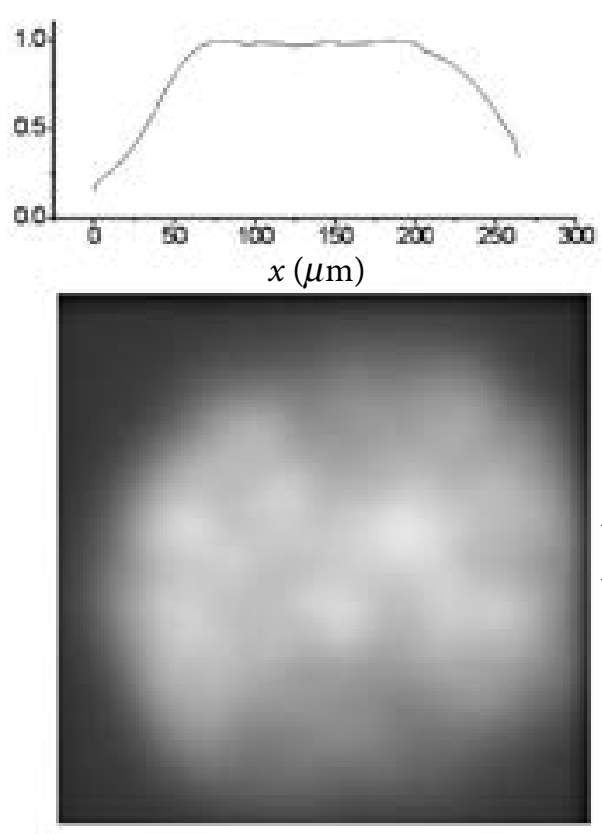

Fig. 1. The near-field intensity distribution of the pump. developed as is common in actively Q-switched lasers with short cavity lifetimes, we can infer the highest Hermite-Gauss mode from the measured average spatial intensity variation period $L=40 \mu \mathrm{m}$. Thereby we can estimate the maximum number of spatial modes required in the modal expansion as $n=4 a / \Lambda \approx 11$, where $a$ is the beam radius [24].

Due to the phase-sensitive nature of the parametric interaction it is important to investigate the coherence properties of the pump beam. We measured the spatial and temporal coherence of the pump in the focal plane by imaging it through a Michelson interferometer with image flipping. The schematic of this experimental arrangement is shown in Fig. 2. In this setup, the waist of the pump was imaged by a $f=100 \mathrm{~mm}$ lens and through the Michelson interferometer, which had a single mirror in one arm, M1, while the two mirrors, M2 and M3, in the other arm provided one additional reflection resulting in an image flipping. The beams from the interferometer arms were superimposed in the plane of the CCD ma- trix, at a small angle, such that the size of the interferometric fringes could be readily resolved. The mirror M1 was mounted on the translation stage, allowing time-domain coherence measurements. A typical CCD image of the pump at zero time delay between the interferometer arms is shown in Fig. 3. The spatial coherence can be characterized by using the spatial distribution of the interferometric fringe visibility function

$$
V\left(r_{N}\right)=\left(I_{\max }-I_{\min }\right) /\left(I_{\max }+I_{\min }\right)
$$

evaluated at the position $r_{N}$ of the Nth fringe. Here $I_{\max }$ and $I_{\min }$ are the maximum and minimum intensities in the fringe, respectfully. Generally, the visibility function is proportional to the degree of coherence, defined as

$$
g\left(r_{1}, r_{2}, \tau\right) \equiv \frac{\left\langle E_{1}^{*}\left(r_{1}, t\right) E_{2}\left(r_{2}, t+\tau\right)\right\rangle}{2 \sqrt{I_{1} I_{2}}},
$$

where $E_{1}\left(I_{1}\right)$ and $E_{2}\left(I_{2}\right)$ are the electric fields (intensities) of the interfering beams, and the brackets

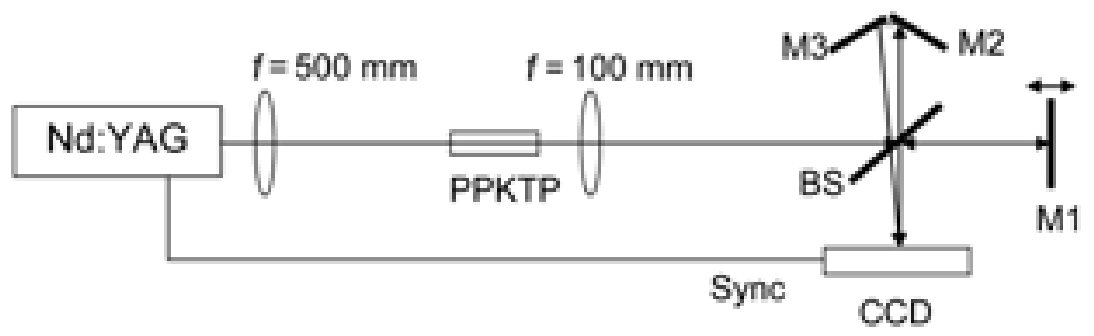

Fig. 2. Michelson interferometer with image flipping for the firstorder correlation measurements. BS, beam splitter; M1-M3, metallic mirrors; CCD, a high framerate CCD camera synchronized with a Nd:YAG Q-switched laser. 


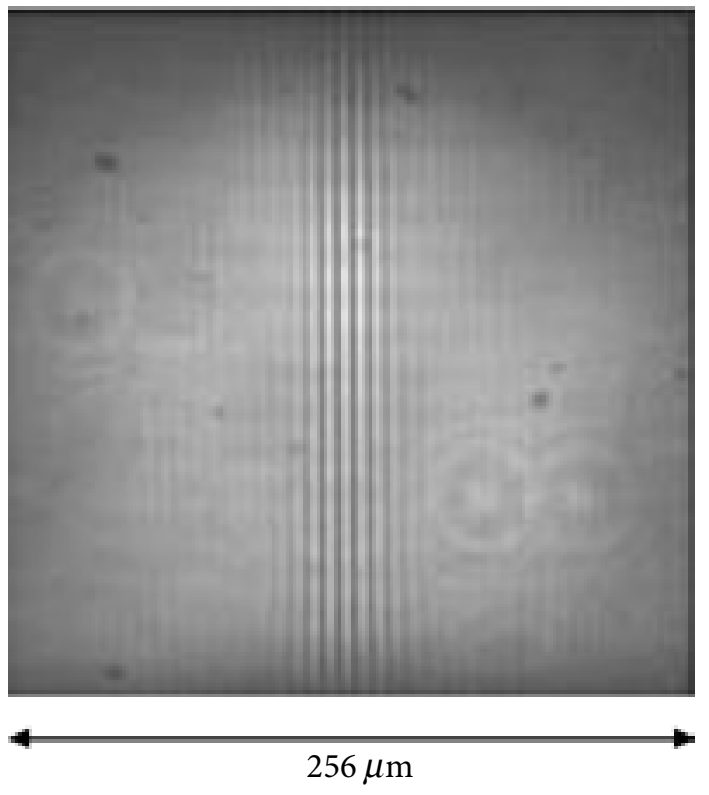

Fig. 3. Spatial correlation of the pump beam at zero delay.

signify the ensemble average. The visibility function measured in our setup,

$$
V(x, \tau)=\frac{2 \sqrt{I_{1} I_{2}}}{I_{1}+I_{2}}|g(x,-x, \tau)|
$$

will give the degree of the first-order coherence along the direction of image flipping. For the radially symmetric beams in our case, this measurement is sufficient to reveal spatial and temporal coherence properties. The measured FWHM spatial coherence radius of the pump beam in the focal plane (hereafter we denote it as the near field) was only about $10 \%$ of the pump waist radius. The evolution of the pump visibility function with time delay is shown in Fig. 4. This data shows the pump
FWHM temporal coherence of $\tau_{\mathrm{pc}}=15$ ps. This coherence time would correspond to the pump frequency bandwidth of about $30 \mathrm{GHz}$ assuming a Gaussian process, which is a typical bandwidth for high-power Q-switched Nd:YAG lasers without seeding or longitudinal mode selection.

The optical parametric generation in PPKTP reached a measurable threshold (about $1 \mu \mathrm{J}$ of the signal energy) at pump energy of around $300 \mu \mathrm{J}$. At pump energy of $405 \mu \mathrm{J}$, the far-field divergence of the OPG signal was about $15 \mathrm{mrad}$, or almost 10-times the diffraction-limited one if we assume that the parametric signal beam waist has the same radius as the pump $(113 \mu \mathrm{m})$. The corresponding far-field signal intensity distribution, shown in Fig. 5(a), was rather smooth with few irregularities. However, the signal near-field intensity distribution, shown for different pump intensities in Fig. 5(b-d), had a very different character. First, the signal near field is composed of beamlets, or parametric gain channels, with the average FWHM radius as small as $7.5 \mu \mathrm{m}\left(1 / \mathrm{e}^{2}\right.$ radius of $12.7 \mu \mathrm{m}$ determined with a standard deviation of $\pm 1.8 \mu \mathrm{m}$ ). Those gain channels were apparently stable within a single pulse but their positions changed randomly from pulse to pulse. Second, the size of the beamlets did not change appreciably with increasing pump power, only their total number increased, eventually, at higher pump powers, filling most of the pump aperture. Assuming that the signal waves in individual channels have perfect spatial coherence, the expected far-field divergence angle on the average would be $16.3 \mathrm{mrad}$ (standard deviation $2.3 \mathrm{mrad}$ ). It corresponds, within the measurement uncertainty, to the experimental value of $15 \mathrm{mrad}$. In that respect one can say

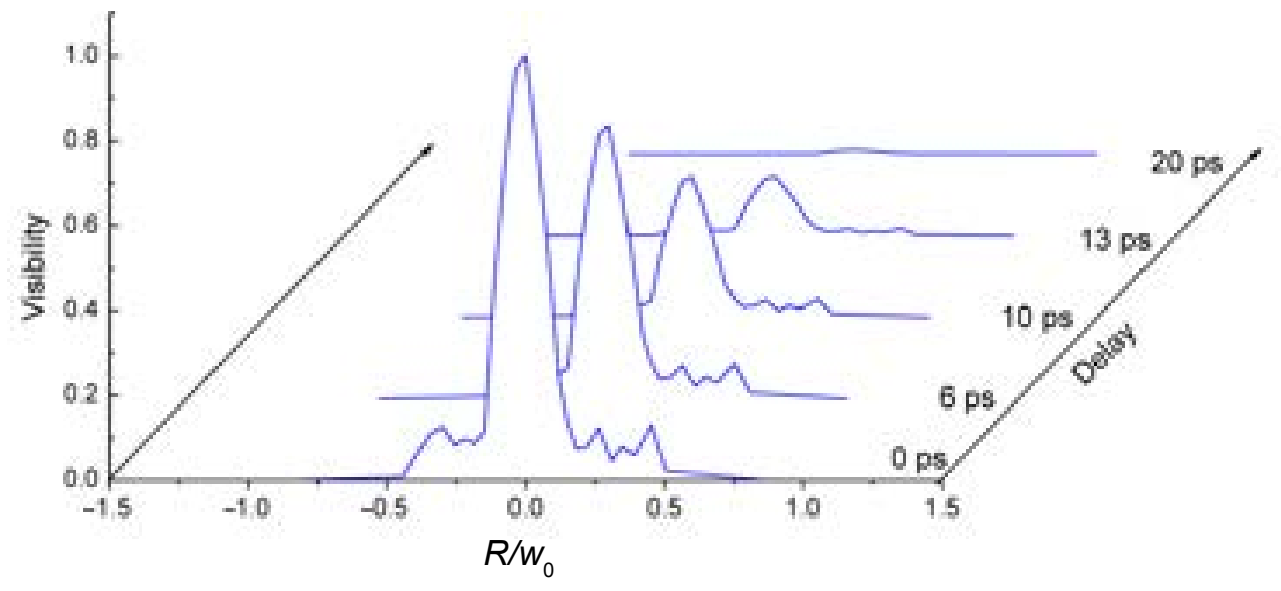

Fig. 4. Dependence of the visibility function of the pump on Michelson delay time. $R / w_{0}$ denotes the ratio of the position in the horizontal plane to the beam radius. 

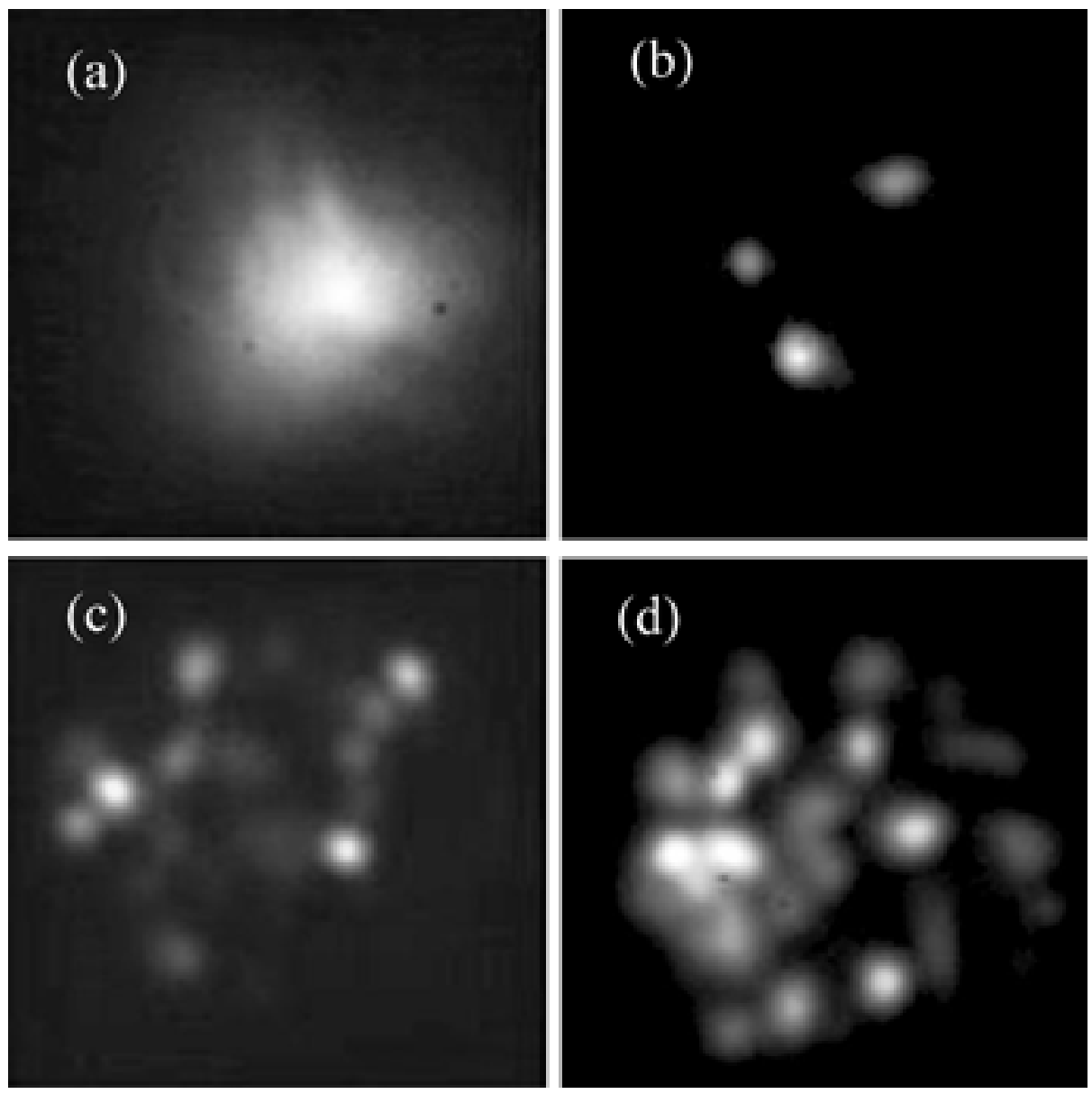

Fig. 5. Single-shot images of the OPG signal: (a) far field at $405 \mu \mathrm{J}$ pump energy; (b-d) near field at different pump energies; (b) at threshold (pump energy $300 \mu \mathrm{J})$; (c) at $360 \mu \mathrm{J}$; (d) at $405 \mu \mathrm{J}$.

that the OPG beam is in fact "diffraction-limited" if we take into account that the diffraction is from individual small-radius beamlets. The assumption that the signal waves are perfectly spatially coherent within the gain channel but completely uncorrelated between different channels has been tested using the same imaging Michelson interferometer with image flipping as described above (see Fig. 2). The OPG near-field and far-field correlations are shown in Fig. 6(a) and 6(b), respec- tively. As can be seen from the near-field image, perfect field correlations are observed only within a single gain channel. In the far field the correlations extend over a much larger area (Fig. 6(b)) as a result of diffraction from narrow gain channels. These observations elucidate the assertion that the OPG signal in the far field is a sum of uncorrelated fields stemming from different beamlets, while the far-field divergence is essentially determined by the diffraction of fully spatially
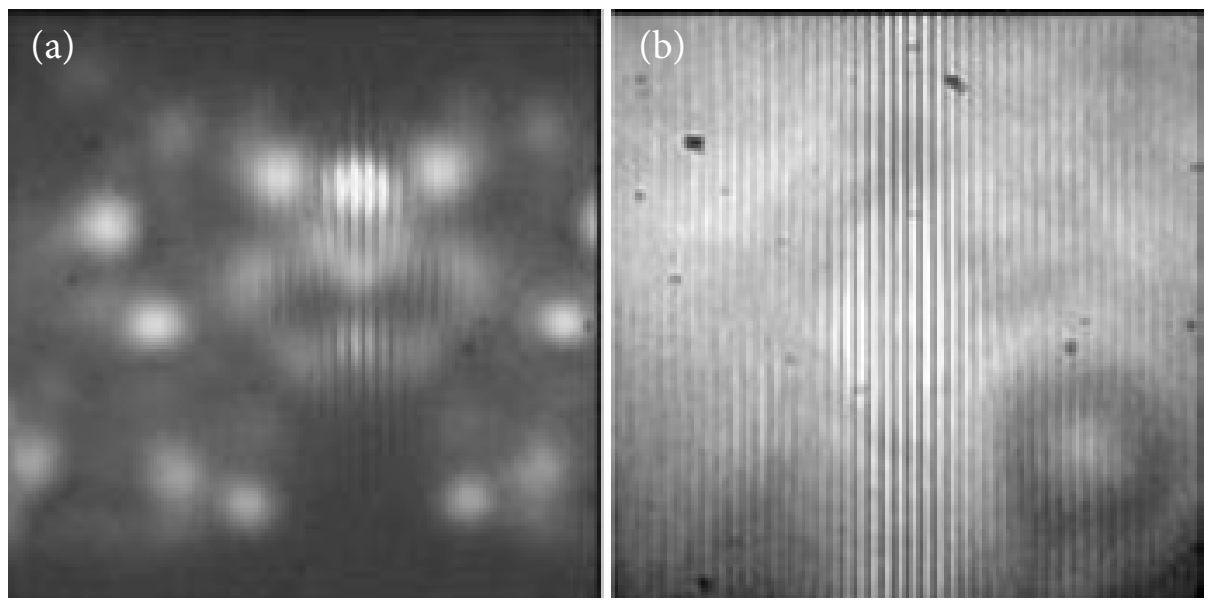

Fig. 6. Spatial correlations of the near field (a) and far field (b) of the OPG beam. 
coherent fields from these individual gain channels. Due to the random pulse-to-pulse distribution of the gain channels, we could only measure the temporal correlation of the OPG signal far field. The measured FWHM of the temporal coherence of the signal was 2 ps, the corresponding frequency bandwidth of $220 \mathrm{GHz}$ assuming the Gaussian process and the spectral bandwidth of $0.3 \mathrm{~nm}$. This bandwidth is very close to the signal spectral bandwidth of $0.31 \mathrm{~nm}$ measured by the time-integrating spectrum analyzer ( $A N D O$ AQ-6315) (see Fig. $7($ a)). The theoretical parametric gain bandwidth at the pump intensity $I_{\mathrm{p}}$ can be evaluated from $\Delta \lambda_{\mathrm{s}}=2 \lambda_{\mathrm{s}}^{2}(\pi c)^{-1}[\mathrm{~g} \ln 2 / L]^{1 / 2}\left|\gamma_{\mathrm{si}}\right|$, where $L$ is the crystal length, $l_{\mathrm{s}}$ is the signal central wavelength, $1 / \gamma_{m j}=1 / v_{g m}-1 / v_{\mathrm{g} j}$ gives the groupvelocity mismatch (GVM) and the derivatives are evaluated at the phase matching point [25]. The coefficient $g=\left(2 \omega_{\mathrm{s}} \omega_{\mathrm{i}} d_{\text {eft }}^{2} I_{\mathrm{p}} / \varepsilon_{0} n_{\mathrm{p}} n_{\mathrm{i}} n_{\mathrm{s}} c^{3}\right)^{1 / 2}$ gives the OPG bandwidth dependence on the pump intensity. For the pump pulse energy of $300 \mu \mathrm{J}$ at the OPG threshold and the effective nonlinear coefficient, $d_{\text {eff }}=10 \mathrm{pm} / \mathrm{V}$, the above relation gives the OPG signal bandwidth of $0.34 \mathrm{~nm}$, fairly close to the experimentally measured value.

Due to the noncollinear interaction and slightly different propagation angles we should expect the central wavelength of each individual signal beamlet to be slightly different. For the same reason, the spectral width of the OPG signal is essentially related to the angle subtended in the nonlinear crystal by the ensemble of beamlets. In order to experimentally verify these assumptions, we used an imaging spectrometer to investigate the spectral properties of the separate signal beamlets. The spectrometer incorporated a 1200 lines/mm diffraction grating and had an arm length of $1 \mathrm{~m}$. The near field of the signal was imaged onto the input slit $(100 \mu \mathrm{m})$ of the spectrometer. The slit was used to select the signal beamlets in the central part of the pump beam. Single-shot spectra were recorded by a CCD camera synchronized with the pump laser. The dispersion of the spectrometer was calibrated by using a temperature-tuned OPO built around the same PPKTP crystal and found to be $0.67 \mathrm{~nm} / \mathrm{mm}$ in the CCD plane for the second diffraction order, giving the nominal spectral resolution of about $0.05 \mathrm{~nm}$. The examples of spectrally resolved signal near-field images generated with different pump pulses are shown in Fig. F(b). The single-shot spectrum typically consists of several peaks with the average spectral width of $0.1 \mathrm{~nm}$, which are distributed over a range of up to $0.8 \mathrm{~nm}$. By taking the spectral average over several CCD frames we obtained a FWHM spectral bandwidth of $0.27 \mathrm{~nm}$. Averaging over a larger number of frames and over the full beam cross-section should give the spectral width equal to the measured spaceand-time-integrated signal bandwidth of $0.31 \mathrm{~nm}$. This measurement clearly demonstrates that separate signal beamlets do indeed have different central wavelengths while the averaged spectrum sets the scale for the temporal coherence of the OPG signal in the far field.

From the standpoint of a simple understanding of the OPG process involving multimode pump beams, the generation would initially start
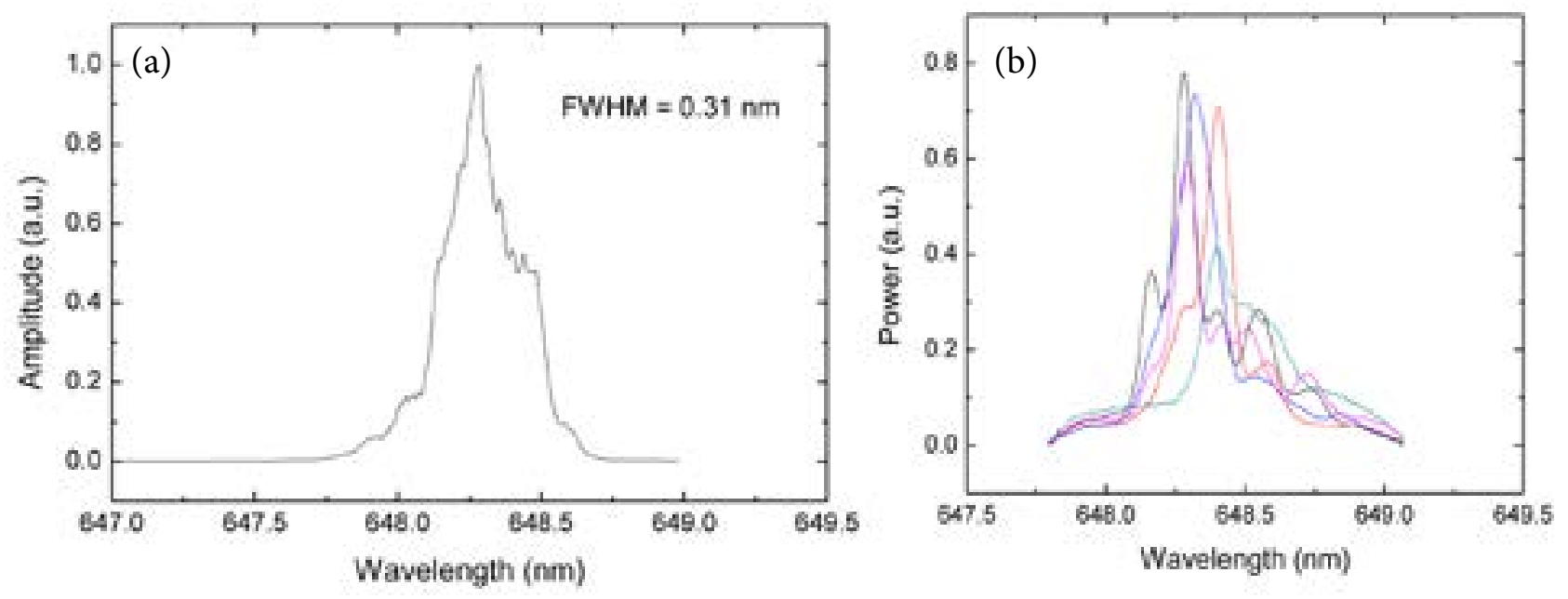

Fig. 7. Time-integrated OPG signal spectrum (a), several realizations of the single-shot OPG signal spectra (b). Each peak in (b) corresponds to an emission from a single parametric gain channel. 
in the local regions of higher pump intensity, and as the parametric amplification progresses, the spatial regions with lower signal intensity will eventually catch up due to higher parametric gain. This picture is reasonable and the only one possible without taking into account effects of spatial and temporal correlations. However, it does not explain why the gain channels observed in OPG have an excellent spatial coherence, why they in fact remain narrower than the spatial modulations in the pump beam and seem to defy diffraction. Moreover, at higher pump powers the local gain in the areas with a larger parametric signal will be lower than in other beam areas, due to the parametric gain decrease with signal power. This should equalize the intensity distribution in the parametric output beam. Clearly this picture, based purely on the local parametric gain and its dependence on the pump intensity distribution, does not explain our experimental observations. Moreover, the spectral width of the signal from a separate gain channel is about three times narrower than what would be expected from the parametric gain expression taking into account GVM.

\subsection{Physical mechanism}

The physical mechanism, which, we believe, is responsible for the formation of the "grainy" parametric intensity pattern in the near field, can be understood by considering the spatial and temporal evolution of spatial and temporal correlations during the parametric interaction in the nonlinear crystal. To illustrate the argument, it is useful to start from the standard nonlinear coupled wave equations describing a spatially and temporally broadband parametric interaction in nonlinear media without the Poynting vector walk-off, as is the case in the quasi-phase matched materials:

$$
\begin{aligned}
& \left(\frac{\partial}{\partial z}-\frac{i}{2 k_{\mathrm{p} z}} \nabla_{\perp}^{2}\right) A_{\mathrm{p}}=\mathrm{i} \kappa A_{\mathrm{s}} A_{\mathrm{i}} \exp (-\mathrm{i} \Delta \mathbf{k z z}) \\
& \left(\frac{\partial}{\partial z}+\frac{1}{\gamma_{\mathrm{ip}}} \frac{\partial}{\partial \tau}-\frac{i}{2 k_{\mathrm{i} z}} \nabla_{\perp}^{2}\right) A_{\mathrm{i}}=\mathrm{i} \kappa A_{\mathrm{p}} A_{\mathrm{s}}^{*} \exp (\mathrm{i} \Delta \mathbf{k z z}),(4) \\
& \left(\frac{\partial}{\partial z}+\frac{1}{\gamma_{\mathrm{sp}}} \frac{\partial}{\partial \tau}-\frac{i}{2 k_{s z}} \nabla_{\perp}^{2}\right) A_{\mathrm{s}}=\mathrm{i} \kappa A_{\mathrm{p}} A_{\mathrm{i}}^{*} \exp (\mathrm{i} \Delta \mathbf{k z z})
\end{aligned}
$$

Here $A_{j}=\left|A_{j}\right| \exp \left(\mathrm{i} \varphi_{j}\right)$ is complex field amplitudes $(j=\mathrm{p}, \mathrm{s}$, $\mathrm{i}$ for the pump, signal and idler, respectively), from the electric field definition: $E_{j}=\sqrt{\omega_{j} / n_{j}} A_{j}\left(r_{\perp}, z, t\right) \exp \left(\mathrm{i}\left(\mathbf{k}_{\mathbf{j}} \mathbf{z}-\omega_{j} t\right)\right)+$ c.c.

The nonlinear coupling coefficient is then $\kappa=d_{\text {eff }} / c \sqrt{\omega_{\mathrm{p}} \omega_{\mathrm{s}} \omega_{\mathrm{i}} / n_{\mathrm{p}} n_{\mathrm{s}} n_{\mathrm{i}}}, k_{j z}$ is the wavevector component along the axial propagation direction and $n_{j}$ represents the refractive indices at central frequencies $\omega_{j}$. $\nabla_{\perp}^{2}$ is the transversal Laplacian, which is needed when handling multi-transversal mode interacting beams, with vastly different diffraction properties. The coupled wave equations are written in the usual coordinate frame moving with the group-velocity of the pump, $\tau=t-z / v_{\mathrm{gp}}$, while the signal and the idler are related to it via appropriate GVM coefficients. The approximation of the non-dispersive propagation of quasi-monochromatic waves becomes insufficient in describing the temporal properties of a broadband parametric device, where the group-velocity walk-off between the interacting waves becomes comparable to the coherence length [26, 27]. Even though the pump in our experiment was in the form of long and relatively narrowband pulses $(\sim 30 \mathrm{GHz})$, the OPG bandwidth is about an order of magnitude larger, so the GVM effects are important. For simplicity we did not include group-velocity dispersion, which would become important for more broadband interactions.

Following [28] it is possible to write formally, from Eq. 4, the coupled wave equations for the first-order correlation functions:

$$
\begin{aligned}
& \frac{\partial g_{\mathrm{p}}\left(r_{1}, r_{2}, r_{1}, \tau_{2}, z\right)}{\partial z}-\frac{\mathrm{i}}{2 k_{\mathrm{p} z}} \nabla_{\perp_{r_{\mathrm{i}}}}^{2} g_{\mathrm{p}} \\
& =\mathrm{i} \kappa\left\langle A_{\mathrm{s}}\left(r_{1}, \tau_{1}, z\right) A_{\mathrm{i}}\left(r_{1}, \tau_{1}, z\right) A_{\mathrm{p}}^{*}\left(r_{2}, \tau_{2}, z\right)\right\rangle, \\
& \frac{\partial g_{\mathrm{i}}\left(r_{1}, r_{2}, r_{1}, \tau_{2}, z\right)}{\partial z}+\frac{1}{\gamma_{\mathrm{ip}}} \frac{\partial g_{\mathrm{i}}}{\partial \tau_{1}}-\frac{\mathrm{i}}{2 k_{\mathrm{i} z}} \nabla_{\perp_{r}}^{2} g_{\mathrm{i}} \\
& =\mathrm{i} \kappa\left\langle A_{\mathrm{p}}\left(r_{1}, \tau_{1}, z\right) A_{\mathrm{s}}^{*}\left(r_{1}, \tau_{1}, z\right) A_{\mathrm{i}}^{*}\left(r_{2}, \tau_{2}, z\right)\right\rangle, \\
& \frac{\partial g_{\mathrm{s}}\left(r_{1}, r_{2}, r_{1}, \tau_{2}, z\right)}{\partial z}+\frac{1}{\gamma_{\mathrm{sp}}} \frac{\partial g_{\mathrm{s}}}{\partial \tau_{1}}-\frac{\mathrm{i}}{2 k_{\mathrm{s} z}} \nabla_{\perp_{r_{\mathrm{i}}} g_{\mathrm{s}}}^{2} \\
& =\mathrm{i} \kappa\left\langle A_{\mathrm{p}}\left(r_{1}, \tau_{1}, z\right) A_{\mathrm{i}}^{*}\left(r_{1}, \tau_{1}, z\right) A_{\mathrm{s}}^{*}\left(r_{2}, \tau_{2}, z\right)\right\rangle .
\end{aligned}
$$

Here the angle brackets signify the ensemble average (involving integration over variables $r_{2}, \tau_{2}$ ), $r \equiv(x, y)$ is the transversal coordinate and the subscripts at Laplacians indicate that they should be evaluated at the transversal position $r_{1}$ while 
the time derivatives are taken with respect to $\tau_{1}$. The right-hand sides of Eq. (5) contain triple correlation functions among the interacting fields. Although here they appear from a totally classical picture, they are reminiscent in the triple correlations found before in the context of evolution of the quantum-mechanical quasi-probability Wigner distribution for OPA [16]. For the case of the undepleted pump the first of Eq. 5 becomes homogeneous. Then the evolution of the signal and idler correlations will be driven by the cross-correlations of these fields and multiplied by the complex pump amplitude. This essentially provides a nonlinear mechanism for diffraction suppression in the parametric interaction. Obviously, Eq. (5) contains the same information as Eq. (4), but it gives a somewhat better insight in the context of the subject of this work.

In order to estimate the importance of the diffraction and GVM in relation to the strength of the nonlinear interaction, it is useful to introduce the characteristic length scales for each relevant effect, $L_{\mathrm{D}}, L_{\mathrm{NL}}, L_{\mathrm{GVM}}$, representing diffraction, nonlinear interaction, and GVM, respectively. It is well known that the field correlation functions as well as time- and space-dependent photon creation and annihilation operators in free propagation obey the wave equations of the form of homogeneous Eq. (5) [28, 29]. During the free propagation through the crystal the areas of initially established correlations, $g_{p}, g_{s}$ and $g_{i}$, would spread in the transversal plane by diffraction and they become separated in time due to the groupvelocity walk-off. The increase of the correlation radius $a_{j}$ due to diffraction areas can then be described by the equivalent solution for the expanding radius of Gaussian beams, namely:

$$
a_{j}(z)=a_{j 0} \sqrt{1+\left[\frac{z \lambda_{j}}{2 \pi n_{j} a_{j 0}^{2}}\right]^{2}} .
$$

Here $\lambda_{j}$ is the wavelength in vacuum. This allows us to define the effective diffraction length for the $j$ th wave as $L_{\mathrm{D}, j}=k_{j} a_{j}^{2}$, which is nothing else but the expression of the equivalent confocal parameter for spatial correlation areas. Taking the measured values of the spatial correlation width and assuming that the initial spatial correlation of the idler wave is equal to that of the signal, we deduce diffrac- tion lengths for the signal and the idler of 2.91 and $0.62 \mathrm{~mm}$, respectively. Due to diffraction, the initial FWHM of the correlation areas of interacting waves should increase to about 105 and $495 \mu \mathrm{m}$ for the signal and the idler fields, respectively, after propagation through the $12 \mathrm{~mm}$ long PPKTP crystal. It seems that the diffracting signal and idler waves can play the role of coherent seeds for the rest of the pump beam. However, due to the uncorrelated phases of the pump field outside the initial correlation radius, as well as the superposition of the uncorrelated parametric fields originating from different initial pump beam areas, the average parametric gain outside the pump correlation radius should be strongly reduced [30]. Consequently, the signal field remains confined within the pump correlation radius where it experiences the maximum gain, giving rise to the beamlet structure in the near-field intensity distribution, as shown in Fig. 5. Indeed, the experimentally determined FWHM radius of $7.5 \mu \mathrm{m}$ of the signal channel is well within the FWHM coherence radius of the pump near field of $11 \mu \mathrm{m}$.

The characteristic nonlinear interaction length can be easily derived from the nonlinear coupled wave equations for the fields (Eq. (4)) by assuming a constant pump and phase-matched interaction of quasi-monochromatic plane waves. This gives an effective nonlinear interaction length as $L_{\mathrm{NL}}=1 / \sqrt{\kappa^{2}\left|A_{\mathrm{p}}\right|^{2}}$. For the experimental parameters at the OPG threshold and using an effective nonlinear coefficient in PPKTP of $d_{\text {eff }}=10 \mathrm{pm} / \mathrm{V}, L_{\mathrm{NL}}$ will be equal to $3.2 \mathrm{~mm}$. Importantly, at the OPG threshold the $L_{\mathrm{NL}}$ approaches the characteristic diffraction length of the signal. At these pump intensities one would expect the parametric gain modifying the diffraction of the parametric waves and essentially forming parametric gain-guiding channels.

The group-velocity walk-off between the parametric waves is one possible mechanism which can affect the temporal correlation time of the parametric waves. The characteristic group-velocity walk-off length, defined as $L_{\mathrm{GVM}}=\tau_{\mathrm{pc}} \gamma_{\mathrm{si}}$ is as long as $48 \mathrm{~mm}$ in the present case, or four-times longer than the nonlinear crystal. Because the $L_{\mathrm{GVM}}$ is substantially longer than the $L_{\mathrm{NL}}$, the GVM has only marginal influence on the temporal coherence of the signal wave. The large $L_{\mathrm{GVM}}$ just informs us that the spectral width (Fourier transform of 
the temporal autocorrelation) of parametric waves will be at most limited by the length of the crystal in correspondence with the experimental findings in the previous subsection.

The spectral width of about $0.1 \mathrm{~nm}$ determined for the signal radiation stemming from a single beamlet should be determined by the noncollinear TWM allowed within the geometrical restrictions of the gain channel. The angular dispersion of the signal caused by noncollinear interactions has been determined to be $4.1 \mathrm{mrad} / \mathrm{nm}$ for this particular PPKTP crystal [31]. This would correspond to the noncollinear internal angle of only $0.41 \mathrm{mrad}$ in the gain channel, which is substantially smaller than the angle of $8.1 \mathrm{mrad}$ expected from the pure linear diffraction of the signal channels inside the PPKTP crystal. On the other hand, this small angle would correspond to the noncollinear deviation of $5 \mu \mathrm{m}$ over the length of the PPKTP crystal. This is actually very close to the measured average geometric radius of the gain channel.

If the range of noncollinear angles would have been determined by the linear diffraction, then we could have expected the signal spectral width of about $2.4 \mathrm{~nm}$. The fact is that we have not observed that a broad signal spectrum once again confirms the physical picture where the parametric waves are confined within the gain channel and the geometry of this channel is determined by the spatial correlation of the pump field. The parametric waves propagating at larger angles experience a much smaller average gain or even a reduction in the amplitude, depending on the relative phases of the waves. Thus the limited spatial coherence of the pump field presents an intriguing mecha- nism which limits both the diffractive spreading of the parametric gain channel and, at the same time, narrows the spectral bandwidth of the parametric waves generated within the beamlets.

One might argue that the beamlets are the result of the filamentation of the optical beams in the nonlinear medium caused by Kerr self-focusing. However, we can rule out this effect in the present case, first, because self-focusing would first affect the pump wave, which has not been observed; second, it has a critical power of about $740 \mathrm{~kW}$ in KTP [32], well above the peak powers used in our experiment ( 120 to $170 \mathrm{~kW}$ ). In order to verify that the diffraction properties of the pump spatial correlation determines the shape and size of the parametric gain channels observed in the near field of the signal wave, we performed a simple experiment where we used a $0.5 \mathrm{~mm}$ wide slit aperture right after the pump focusing lens in order to induce an additional diffraction in the horizontal plane. The parametric signal beamlets generated with this pump acquired an asymmetric elliptical shape with a long axis of the ellipses aligned in the direction of a larger diffraction as shown in Fig. 8 .

\section{Coherence enhancement in parametric devices}

Based on the understanding of the near-field structure of the OPG signal, we can devise ways to enhance the coherence properties and brightness of parametric devices. For instance, the signal coherence time can be increased by selecting parallel parametric gain channels and suppressing noncollinear parametric interactions. The OPO cavity feedback can perform this type of
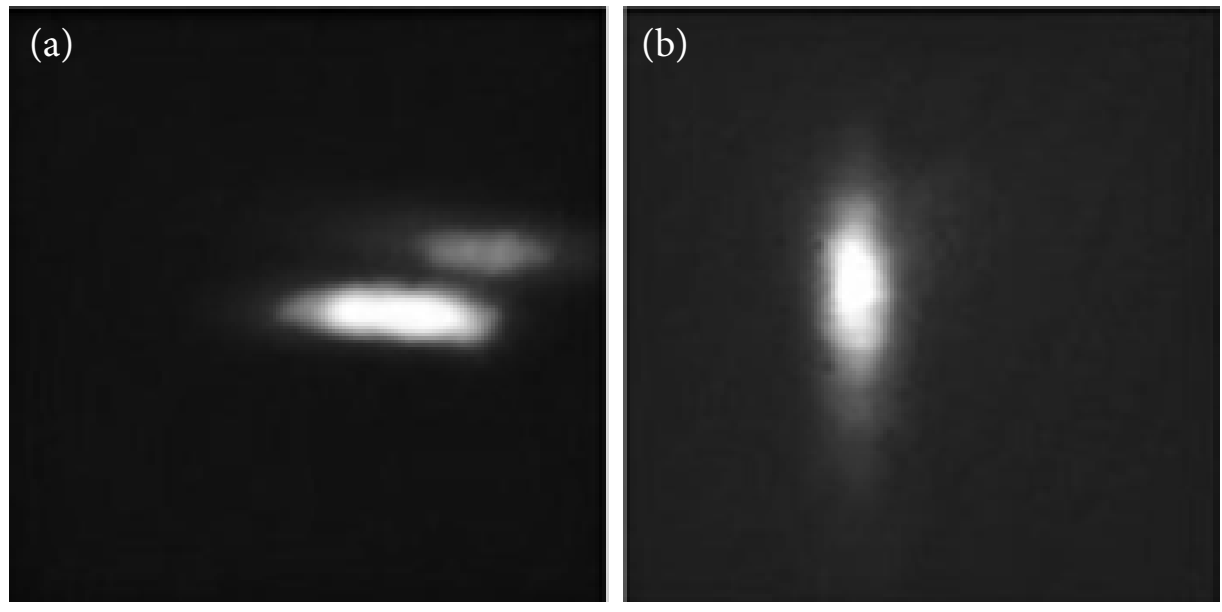

Fig. 8. Near-field (a) and far-field (b) intensities of the signal in an OPG pumped with the pump beam with predominant diffraction in the horizontal plane. 
directional gain selection. To prove this, we constructed a simple $3 \mathrm{~cm}$-long OPO cavity around the PPKTP crystal by adding two plane mirrors with the reflectivity of 99 and $50 \%$ at the signal wavelength and high transmission at the pump and idler wavelengths. The pump focusing conditions were the same as in the OPG case. Operating the OPO at 2.5 times above the threshold of $78 \mu \mathrm{J}$, the signal coherence time increased to $6.2 \mathrm{ps}$ which corresponds to the spectral bandwidth of $0.1 \mathrm{~nm}$, i.e. approximately the spectral extent of a single signal channel. The spatial correlation width of the OPO signal in the near-field, on the other hand, was $0.32 \times w_{s}$, where $w_{s}$ is the signal near-field radius (see Fig. 9). Notably, the spatial coherence of the OPO signal is already threetimes higher than that measured for the pump beam. However, the fact that the signal field originates from an ensemble of largely uncorrelated gain channels puts a limit on the spatial coherence which results in a non-diffraction-limited beam.

From the development above it is clear that in order to increase further the spatial coherence of the parametric field, the multimode pump has to contain a single highly correlated intensity peak in the near field. Multiple-pump beam interference in the near field automatically selects mutually coherent parts of the pump field. In cylindrical symmetry, the intensity pattern of this interference takes the shape of the distribution corresponding to the zeroth-order Bessel function. These patterns, commonly called Bessel-Gauss beams, can be thought of as a superposition of Gaussian beams arranged on a cone [33]. This approach has an important advantage because the interference automatically selects the coherent parts of pump beams and, at the same time, produces regular maxima in the pump intensity distribution, where the parametric gain preferentially occurs. This superposition produces a sharp and extended "nondiffracting" intensity peak along the cone axis so that the parametric gain channel is ideally located along a single line.

Bessel-Gauss beams are most efficiently produced by axicon prisms where the complete power of the pump can be utilized. We constructed a Bessel-Gauss pumped PPKTP OPG by replacing the pump focusing lens with an axicon prism with an apex angle of about $178^{\circ}$. The same multimode pump beam was used for excitation. The nearfield distribution of the OPG signal consisted of a single smooth Gaussian beam (see Fig. 10(a)). Imaging the signal beam through the Michelson interferometer with image flipping revealed that the signal in the near field was perfectly spatially coherent (see Fig. 10(b)), which ensured a diffraction-limited far-field divergence. Due to a relatively broad parametric gain channel $\left(1 / \mathrm{e}^{2}\right.$ intensity radius of $59 \mu \mathrm{m}$ ) the OPG signal spectrum was broadened by noncollinear parametric interactions to about $0.17 \mathrm{~nm}$, which corresponds well with the measured temporal coherence time of 3.5 ps. As the Bessel-Gauss-pumped OPG contains only one gain channel in the near field, a simple OPO cavity feedback is very effective in narrowing the signal spectrum. Indeed, the signal from a short $(3 \mathrm{~cm})$ cavity OPO with both planeplane mirrors, pumped by the Bessel-Gauss beam, had perfect spatial coherence and temporal coherence time of $26 \mathrm{ps}$, which exceeded that of the pump.
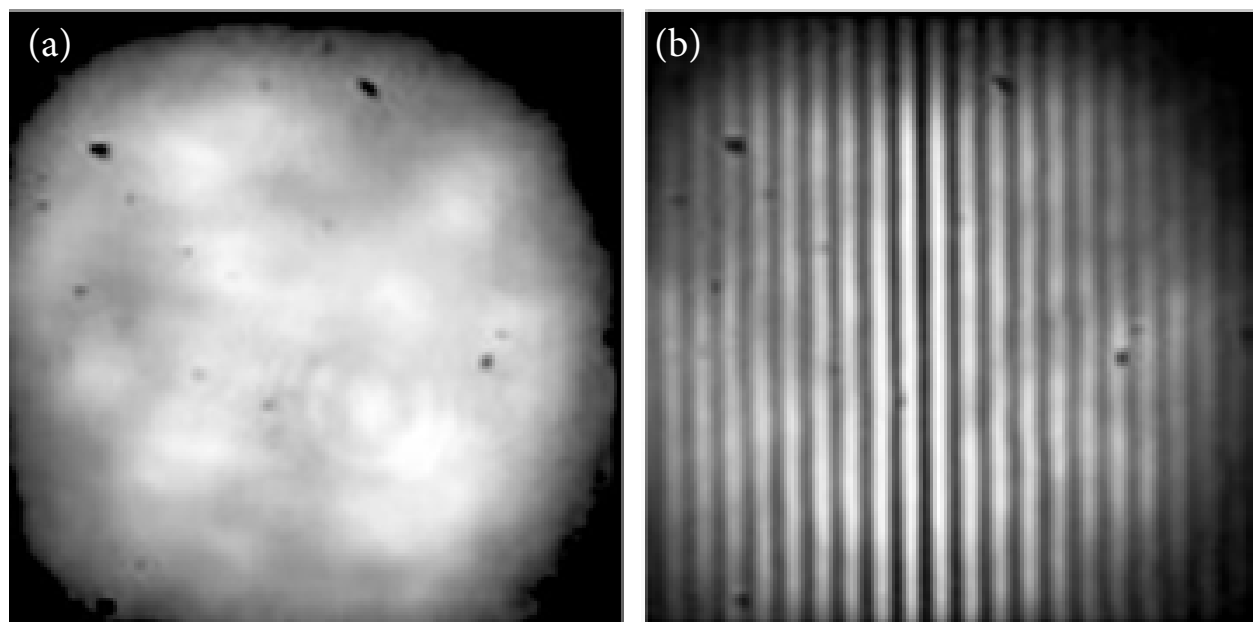

Fig. 9. Intensity images of a short-cavity OPO pumped by a multimode beam. Near-field signal (a) and near-field spatial correlation (b). 

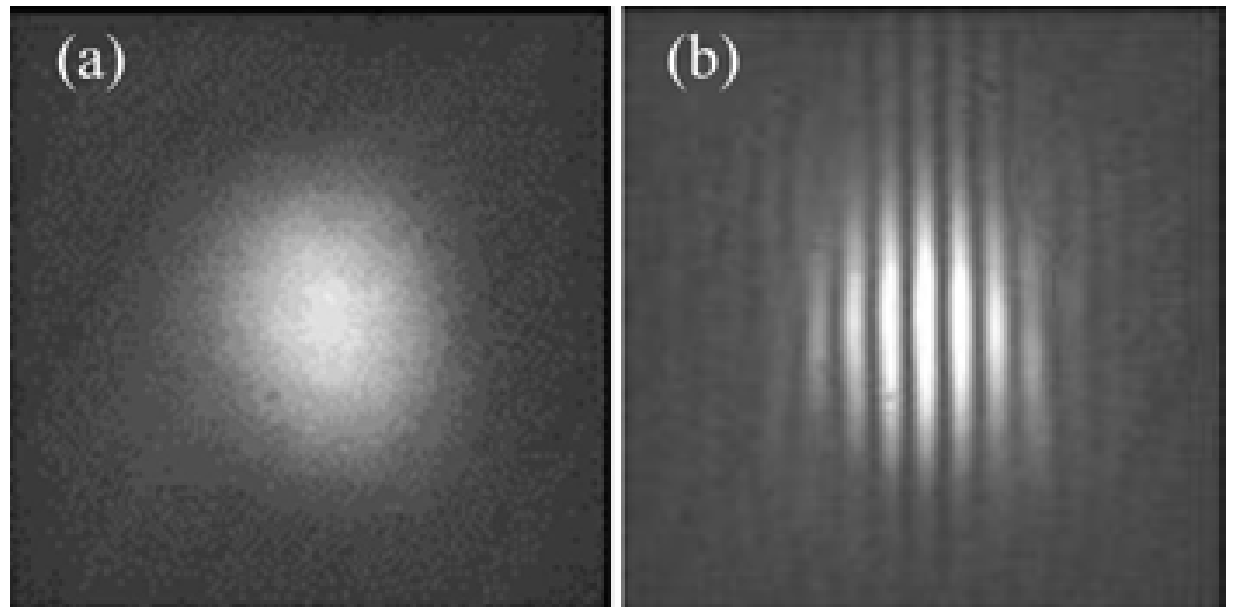

Fig. 10. Signal in the Bessel-Gauss beam pumped OPG: (a) near-field intensity distribution, (b) near-field spatial correlation obtained by imaging Michelson interferometer with image reversal.

\section{Conclusions}

We have shown that the interplay of the coherence properties of a multi-transversal mode pump wave and parametric waves leads to a structured intensity distribution in the near field of the signal wave. More specifically, this structuring in optical parametric generators, seeded by quantum noise and thermal excitations, occurs when the characteristic nonlinear interaction length $L_{\mathrm{NL}}$ becomes close to the characteristic diffraction length of the parametric signal, $L_{D}$. In turn, this diffraction length is determined by the area of spatial correlation of the multi-transversal mode pump. The nonlinear interaction then effectively suppresses the diffraction within the parametric gain channels, a mechanism akin to that in formation of spatial solitons. The signal radiation within each of the parametric gain channels maintains excellent spatial coherence. Correspondingly, the spectral width of the radiation generated within the gain channel is primarily determined by the transverse degree of freedom for the wavevectors within the geometry of the channel. It was found that the simplest physical picture which would explain the experimental observations is in terms of the nonlinear evolution of pump, signal and idler correlation functions. The nonlinear coupled wave equations for the correlation functions include the triple correlations of interacting fields. In the future, it would be interesting to develop a fully quantum-mechanical picture for the nonlinear evolution of the spatio-temporal moments for coherent and mixed states.

Such structuring of the parametric gain in the nonlinear medium obtained with a partially spatially coherent pump is most easily observed in highly nondegenerate TWM interactions, where the diffraction of the signal and idler are very different. Importantly though, these insights give a very useful recipe of how to change the spatial and temporal coherence properties of the radiation generated in parametric devices. In particular, we demonstrated this by employing conical beam pumping using partially-coherent multimode Q-switched laser radiation.

By giving a rather intuitive physical picture of the emergence of coherent parametric radiation in the TWM interaction seeded by uncorrelated noise and pumped by partially coherent beams, we left aside a practical question of how efficient this process of "coherence distillation" can be. A simplistic answer, and one which does not violate the second law of thermodynamics, could be that the efficiency should scale as a square of the ratio of the pump spatial coherence radius to the pump beam radius. However, at least for Bessel-Gauss beam pumping this is not the case and the efficiency could be much higher [22] due to the fact that one of the parametric waves is quickly removed from the central peak region by the requirements of momentum conservation. Therefore, the signal can be further amplified along the central peak of the Bessel distribution without phase constraints.

\section{References}

[1] J.E. Bjorkholm, Some effects of spatially nonuniform pumping in pulsed optical parametric oscillators, IEEE J. Quantum Electron. 7, 109-118 (1971).

[2] M. Eichhorn, G. Stöppler, M. Schellhorn, K.T. Zawilski, and P.G. Schunemann, Gaussian- versus 
flat-top-pumping of a mid-IR ZGP RISTRA OPO, Appl. Phys. B 108, 109-115 (2012).

[3] M. Peltz, U. Bäder, A. Borsutzky, R. Wallenstein, J. Hellström, H. Karlsson, V. Pasiskevicius, and F. Laurell, Optical parametric oscillators for high pulse energy and high average power operation based on large aperture periodically poled KTP and RTA, Appl. Phys. B 73, 663-670 (2001).

[4] A. Zukauskas, N. Thilmann, V. Pasiskevicius, F. Laurell, and C. Canalias, $5 \mathrm{~mm}$ thick periodically poled Rb-doped KTP for high energy optical parametric frequency conversion, Opt. Mater. Express 1, 201 (2011).

[5] H. Ishizuki and T. Taira, High-energy quasiphase-matched optical parametric oscillation in a periodically poled $\mathrm{MgO}: \mathrm{LiNbO}_{3}$ device with a $5 \mathrm{~mm} \times 5 \mathrm{~mm}$ aperture, Opt. Lett. 30, 29182920 (2005).

[6] H. Ishizuki and T. Taira, High energy quasi-phase matched optical parametric oscillation using $\mathrm{Mg}$ doped congruent $\mathrm{LiTaO}_{3}$ crystal, Opt. Express 18, 253-258 (2010).

[7] L.R. Marshall, A. Kaz, and O. Aytur, Multimode pumping of optical parametric oscillators, IEEE J. Quantum Electron. 32, 177-182 (1996).

[8] R. Graham and H. Haken, The quantum-fluctuations of the optical parametric oscillator. I, Zeitschrift für Phys. 210, 276-302 (1968).

[9] S.T. Wong, T. Plettner, K.L. Vodopyanov, K. Urbanek, M. Digonnet, and R.L. Byer, Self-phaselocked degenerate femtosecond optical parametric oscillator, Opt. Lett. 33, 1896-1898 (2008).

[10]S.C. Lyons, G.L. Oppo, W.J. Firth, and J.R.M. Barr, Beam-quality studies of nanosecond singly resonant optical parametric oscillators, IEEE J. Quantum Electron. 36, 541-549 (2000).

[11]J.P. Gordon, L.R. Walker, and W.H. Louisell, Quantum statistics of masers and attenuators, Phys. Rev. 130, 806-812 (1963).

[12]R. Graham, Photon statistics of the optical parametric oscillator including the threshold region, Zeitschrift für Phys. 211, 469-482 (1968).

[13]B.R. Mollow and R.J. Glauber, Quantum theory of parametric amplification. II, Phys. Rev. 160, 1097-1108 (1967).
[14]L.A. Wu, H.J. Kimble, J.L. Hall, and H. Wu, Generation of squeezed states by parametric down conversion, Phys. Rev. Lett. 57, 2520-2523 (1986).

[15]R. Graham, Theory of cross-correlation of signal and idler in parametric oscillators, Phys. Lett. A 32, 373-374 (1970).

[16]P.D. Drummond and P. Kinsler, Triple correlations in non-degenerate parametric oscillators, Quantum Semiclass. Opt. 7, 727-741 (1995).

[17]A. Picozzi and M. Haelterman, Parametric threewave soliton generated from incoherent light, Phys. Rev. Lett. 86, 2010-2013 (2001).

[18]G. Strömqvist, V. Pasiskevicius, C. Canalias, P. Aschieri, A. Picozzi, and C. Montes, Temporal coherence in mirrorless optical parametric oscillators, J. Opt. Soc. Am. B 29, 1194 (2012).

[19]G. Strömqvist, V. Pasiskevicius, C. Canalias, and C. Montes, Coherent phase-modulation transfer in counterpropagating parametric down-conversion, Phys. Rev. A 84, 23825 (2011).

[20]C. Canalias and V. Pasiskevicius, Mirrorless optical parametric oscillator, Nat. Photonics 1, 459462 (2007).

[21]A. Piskarskas, V. Smilgevičius, and A. Stabinis, Optical parametric oscillation excited by an incoherent conical beam, Opt. Commun. 143, 72-74 (1997).

[22] V. Pasiskevicius, H. Karlsson, J.A. Tellefsen, F. Laurell, R. Butkus, A. Piskarskas, V. Smilgevicius, and A. Stabinis, Singly resonant optical parametric oscillator in periodically poled $\mathrm{KTiOPO}_{4}$ pumped by a Bessel beam, Opt. Lett. 25, 969-971 (2000).

[23]H. Karlsson and F. Laurell, Electric field poling of flux grown $\mathrm{KTiOPO}_{4}$, Appl. Phys. Lett. 71, 34743476 (1997).

[24]A.E. Siegman, Lasers (University Science Books, 1986).

[25]C. Manzoni and G. Cerullo, Design criteria for ultrafast optical parametric amplifiers, J. Opt. 18, 103501 (2016).

[26]W.J.A. Russel, J. Gehr, R.L. Schmitt, and A.V. Smith, Beam tilt and angular dispersion in broad-bandwidth, nanosecond optical parametric oscillators, J. Opt. Soc. Am. B 16, 1525-1532 (1999). 
[27]G. Arisholm, Quantum noise initiation and macroscopic fluctuations in optical parametric oscillators, J. Opt. Soc. Am. B 16, 117-127 (1999).

[28]L. Mandel and E. Wolf, Optical Coherence and Quantum Optics (Cambridge University Press, 1995).

[29]M. Kolobov, The spatial behavior of nonclassical light, Rev. Mod. Phys. 71, 1539-1589 (1999).

[30]A. Picozzi, C. Montes, and M. Haelterman, Coherence properties of the parametric threewave interaction driven from an incoherent pump, Phys. Rev. E 66, 56605 (2002).
[31]V. Pasiskevicius, H. Karlsson, F. Laurell, R. Butkus, V. Smilgevicius, and A. Piskarskas, Highefficiency parametric oscillation and spectral control in the red spectral region with periodically poled $\mathrm{KTiOPO}_{4}$, Opt. Lett. 26, 710-712 (2001).

[32]M. Sheik-Bahae and M. Ebrahimzadeh, Measurements of nonlinear refraction in the secondorder materials $\mathrm{KTiOPO}_{4}, \mathrm{KNbO}_{3}$, beta- $\mathrm{BaB}_{2} \mathrm{O}_{4}$, and $\mathrm{LiB}_{3} \mathrm{O}_{5}$, Opt. Commun. 142, 294-298 (1997).

[33]V. Bagini, F. Frezza, M. Santarsiero, G. Schettini, and G.S. Spagnolo, Generalized Bessel-Gauss beams, J. Mod. Opt. 43, 1155-1166 (1996).

\title{
DAUGIAMODŽIAIS LAZERIAIS KAUPINAMŲ OPTINIŲ PARAMETRINIŲ DAŽNIO KEITIKLIU SPINDULIUOTĖS ERDVINIS IR LAIKINIS KOHERENTIŠKUMAS
}

\author{
V. Pašiškevičius ${ }^{a}$, V. Smilgevičius ${ }^{b}$, R. Butkus ${ }^{b}$, R. Coetzee ${ }^{a}$, F. Laurell ${ }^{a}$ \\ ${ }^{a}$ Karališkasis technologijos institutas, Stokholmas, Švedija \\ ${ }^{\mathrm{b}}$ Lazeriniu tyrimu centras, Vilnius, Lietuva
}

\section{Santrauka}

Darbe tiriamas erdvinio ir laikinio koherentiškumo atsiradimas ir vystymasis neišsigimusio antros eiles parametrinio šviesos generavimo procesuose, realizuotuose feroelektriniuose dariniuose, kurie kaupinami daugiamodžių lazeriụ spinduliuote. Darbe parodoma, kad šiomis sąlygomis, kurios yra aktualios praktiniams taikymams, parametrinès spinduliuotès erdvinis skirstinys kristale užgimsta kaip tarpusavyje nekoreliuotų parametrinès spinduliuotès gijų ansamblis, kur paviené tos spinduliuotès gija turi visišką erdvinị koherentišku- mą. Mažas kaupinimo erdvinio koherentiškumo spindulys, būdingas didelès energijos daugiamodžių lazerių spinduliuotei, lemia parametrinio stiprinimo giju skersinius matmenis ir padeda apriboti tiesines difrakcijos sukeliamą parametrinès spinduliuotès gijų išplitimą. Remdamiesi šiais pastebejjimais mes eksperimentiškai pademonstravome, kad manipuliuojant daugiamodžio kaupinimo lauko erdvinemis koreliacijomis netiesiniame kristale įmanoma padidinti generuojamos parametrinès spinduliuotės erdvinị ir laikinị koherentiškumą. 